\title{
Encapsulation in Polysulfone/Polycarbonate/Impact Modifier Blends
}

\author{
Jong Tae Yoon, Won Ho Jo, Ick Hwan Kwon, ${ }^{*}$ \\ Sang Cheol LeE, ${ }^{* *}$ and Moo Sung LeE***,† \\ Department of Fiber and Polymer Science, Seoul National University, Seoul 151-742, Korea
$* R \& D$ Center, Cheil Industries Inc., Euiwang 437-010, Korea
$* *$ Department of Polymer Science and Engineering, Kum-Oh National University
of Technology, Kumi, Korea
}

(Received August 31, 1998)

\begin{abstract}
Ternary blends of polysulfone (PSF)/polycarbonate (PC)/Impact modifiers (IM) were prepared to improve the mechanical properties of PSF/PC binary blends. Two different core-shell impact modifiers, methyl methacrylate-butadieneethylacrylate (MBE) and acrylonitrile butadiene styrene (ABS) terpolymers, were used. Morphology of the blends was very dependent on the matrix type but independent of the IM type. For PSF matrix PC tends to encapsulate the IM. Whereas, for PC matrix, the IM tends to locate in PC matrix and thus two distinct phases of PSF and IM are observed. The encapsulation of PC onto the IM in PSF matrix was verified from the model blends of PSF/PC/SAN24 and PSF/PC/PMMA, where SAN24 and PMMA are corresponding to the shell of the IM, respectively. Impact strengths of the blends with the IM were slightly increased compared to PSF/PC binary blends, but not significantly. This may be the result of poor PSF/PC interfaces, which are valid from their immiscibility.

KEY WORDS Polymer Blends/Encapsulation/Toughening/Polysulfone / Polycarbonate / Core-Shell Impact Modifier /
\end{abstract}

Polysulfone (PSF) is one of engineering plastics, which has transparency, heat resistance and outstanding mechanical properties. However, its high softening point and melt viscosity at processing temperature in the range of $320-410 \mathrm{C}$ request robust processing equipment. ${ }^{1}$ Polycarbonate (PC) is also an important engineering polymer used in various applications, because of its balanced properties including optical clarity and toughness. However, it has some problems such as notch sensitivity and liability to crack. These disadvantages have been overcome through blending with other polymers such as acrylonitrile-butadiene-styrene (ABS), ${ }^{2}$ poly(butylene terephthalate). ${ }^{3}$

Blends of PSF and PC may compensate the disadvantages of each other and be a candidate for excellent engineering plastic alloy because they may offer a new materials with higher fluidity of PC with minimum decrease in the high performance of PSF. However, previous studies on PSF/PC blends have reported that the pair is completely immiscible, which is verified from the two distinct composition-independent glass transition temperatures. ${ }^{4.5}$ Kohlman et al. have also reported that PSF/PC blends become more brittle and more notchsensitive with the addition of PSF and the impact strength of the blends decreased drastically only with slight addition of PSF. ${ }^{6}$ It is known that the compatibility between PSF and PC is not improved although wide range of mixing conditions is applied.

In this study, we prepare ternary blends of PSF, PC, and core-shell impact modifiers (IM) to enhance mechanical properties of PSF/PC blends because the addition of IM has been accepted as an effective way to improve the impact strength of brittle polymers and alloys. ${ }^{7.8}$ Especially, we will discuss how the relative ratio of PSF and PC affect to the morphology and mechanical properties of the blends.

\footnotetext{
+ To whom correspondence should be addressed.
}

\section{EXPERIMENTAL}

\section{Materials}

All polymers used in this study are commercially available. The source, molecular weight, and other characteristics of the polymers are listed in Table I. Methyl methacrylate-butadiene ethylacrylate (MBE), ABS, and poly(methyl methacrylate) (PMMA) are obtained in powder form and the others in pellet form. PSF is an Ultrason S 2010 of BASF and is dried at $140^{\circ} \mathrm{C}$ for at least $4 \mathrm{~h}$ before mixing. PC is a Makrolon 2800 of Bayer and is used after drying at $120^{\circ} \mathrm{C}$ for at least $4 \mathrm{~h}$. MBE is an EXL-2602 obtained from Kureha Chemicals. The shell of MBE is covered with PMMA and its core material is butadiene-ethylacrylate copolymers. ABS, which has the shell of graft styrene-acrylonitrile (SAN) copolymers onto butadiene core, has the rubber particle diameter of $0.3 \mu \mathrm{m}$. The acrylonitrile (AN) content in the grafted SAN copolymers is $24 \mathrm{wt} \%$. The plot of complex viscosity $\left(\eta^{*}\right) v s$. frequency $(\omega)$ at a mixing temperature, $270^{\circ} \mathrm{C}$, for the polymers used in this study is given in Figure 1. The data are measured using a Rheometrics Mechanical Spectrometer (RMS 800) with cone and plate mode.

Table I. Characteristics of materials used in this study

\begin{tabular}{|c|c|c|c|c|c|}
\hline \multirow{2}{*}{ Polymer } & \multirow{2}{*}{ Source } & \multirow{2}{*}{$\frac{M_{w}}{\mathrm{~g} \mathrm{~mol}^{-1}}$} & \multirow{2}{*}{ PDI } & \multirow{2}{*}{$T_{\mathrm{g}} /{ }^{\circ} \mathrm{C}$} & \multirow{2}{*}{$\frac{\left|\eta^{*}\right|^{a}}{\operatorname{Pas}}$} \\
\hline & & & & & \\
\hline PSF & BASF & 52000 & 1.63 & 195 & 3057 \\
\hline PC & Bayer & 51000 & 1.82 & 153 & 1416 \\
\hline $\mathrm{ABS}^{\mathrm{b}}$ & Cheil Industries & - & - & - & - \\
\hline $\mathrm{MBE}^{\mathrm{c}}$ & Kureha Chemicals & - & - & - & - \\
\hline SAN24 & Cheil Industries & 97000 & 1.86 & 112 & 234 \\
\hline PMMA & LG Chemicals & 93000 & 1.58 & 115 & 176 \\
\hline
\end{tabular}

${ }^{a}$ Measured using a Rheometrics RMS 800 at a shear rate of $40 \mathrm{~s}^{-1}$ and $270 \mathrm{C}$. ${ }^{\mathrm{b}} 60 \mathrm{wt} \%$ rubber (butadiene) content. ${ }^{\mathrm{c}} 80 \mathrm{wt} \%$ rubber (butadiene ethylacrylate) content. 


\section{Mixing}

Ternary blends of PSF/PC/IM were prepared with a twin screw extruder with a diameter of $32 \mathrm{~mm}$ and $\mathrm{L} / \mathrm{D}$ ratio of 28 . Temperature was set at $270^{\circ} \mathrm{C}$ and the screw revolution of $250 \mathrm{rpm}$ was applied. Ternary blends of

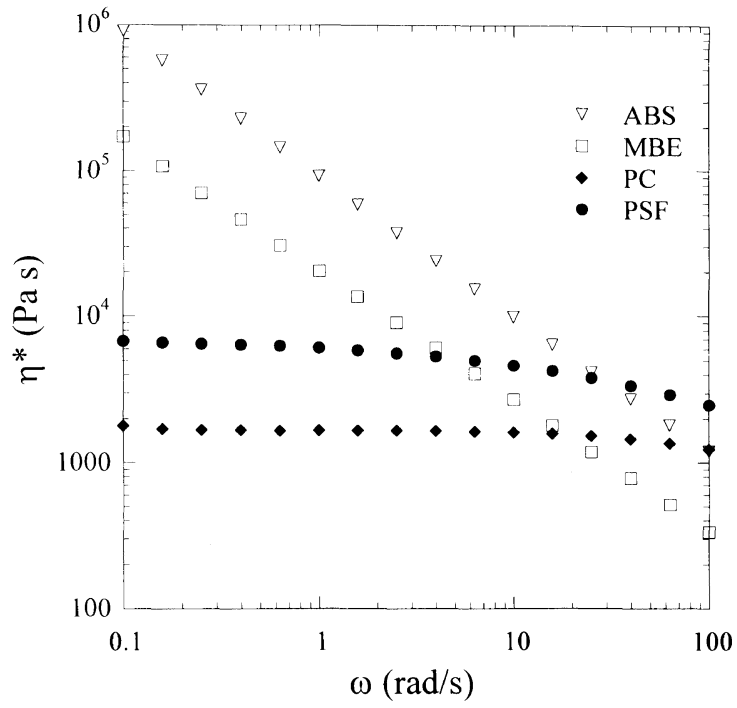

Figure 1. Complex viscosity $\left(\eta^{*}\right) v s$. frequency $(\omega)$ for PSF $(\bullet)$, PC $(\diamond)$, ABS $(\nabla)$, and $\mathrm{MBE}(\square)$ at $270^{\circ} \mathrm{C}$.
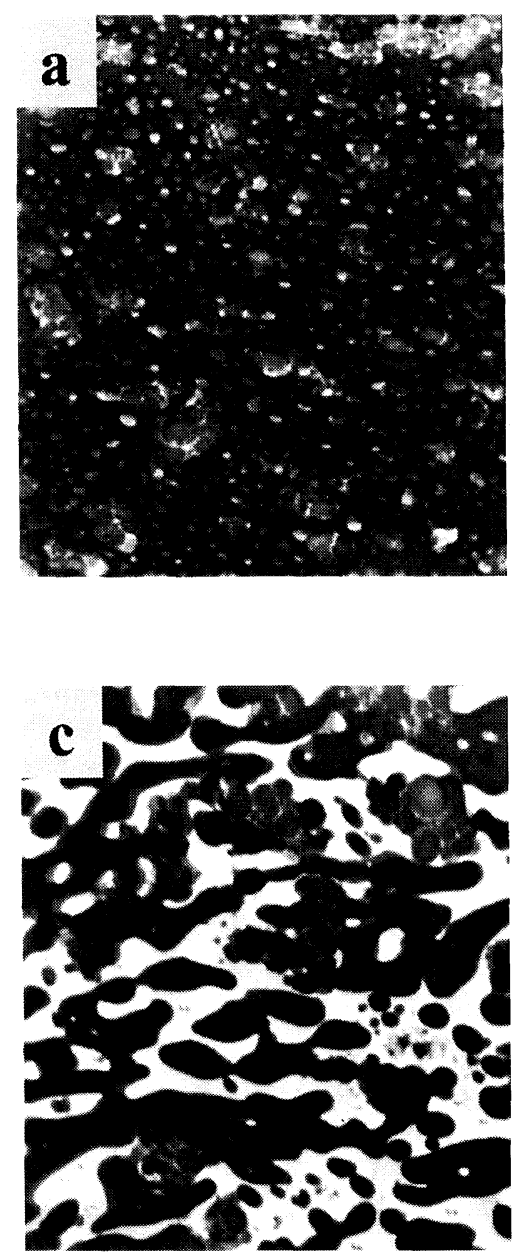

$\mathrm{PSF} / \mathrm{PC} / \mathrm{SAN} 24$ and $\mathrm{PSF} / \mathrm{PC} / \mathrm{PMMA}$ were also prepared as model systems because SAN24 and PMMA correspond to the shells of $\mathrm{ABS}$ and $\mathrm{MBE}$, respectively. A Haake Rheomix 600 batch mixer with roller blades, operating at $270^{\circ} \mathrm{C}$ for $10 \mathrm{~min}$, was used for preparing the model blends.

\section{Morphological Observation}

Blend morphology was obtained using a JEOL JEM 2000EXII transmission electron microscope (TEM), operated at an accelerating voltage of $100 \mathrm{kV}$. Ultrathin sections of $50 \mathrm{~nm}$ thickness were microtomed at room temperature with a Reichert-Jung ultracut microtome. To give a contrast between phases a double staining method was applied. ${ }^{9}$ The microtomed sections were first stained for $48 \mathrm{~h}$ in a water solution of $2 \mathrm{wt} \%$ osmium tetraoxide $\left(\mathrm{OsO}_{4}\right)$ and then stained again for $15 \mathrm{~min}$ with the vapor of an aqueous solution of $0.6 \mathrm{wt} \%$ ruthenium tetraoxide $\left(\mathrm{RuO}_{4}\right)$.

\section{Impact Test}

Impact strengths of blends were measured using an Izod impact tester. After dried at $120^{\circ} \mathrm{C}$ for $4 \mathrm{~h}$ extruded pellets were injection-molded at $270 \mathrm{C}$ for impact test. The mold surface temperature was kept to be $80^{\circ} \mathrm{C}$. The specimens were notched and tested according to ASTM
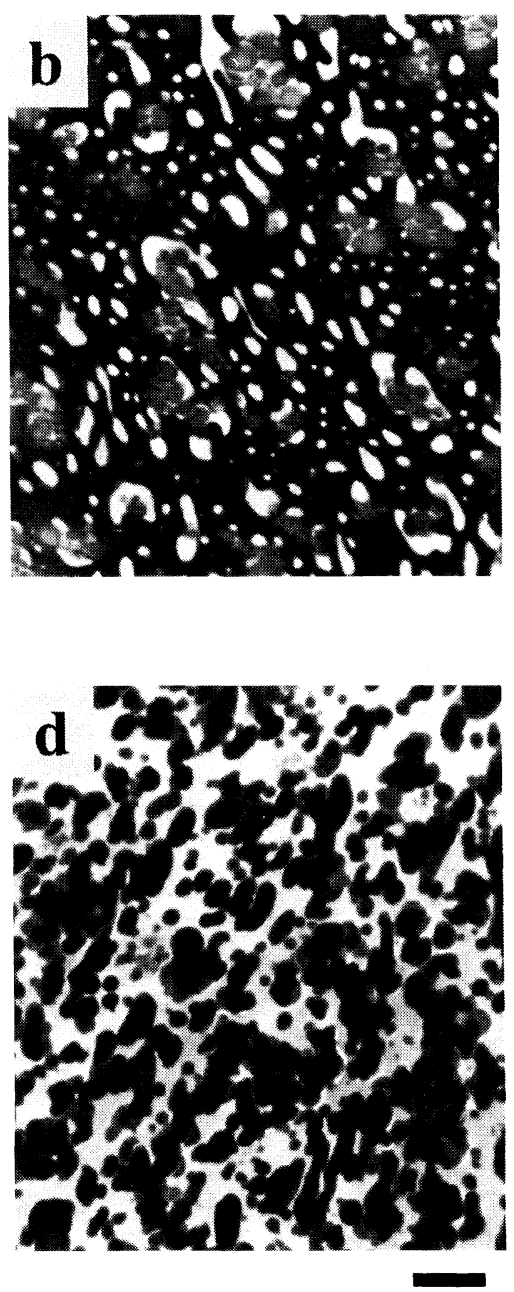

$1 \mu \mathbf{m}$

Figure 2. TEM photographs of PSF/PC/ABS blends with $15 \mathrm{wt} \%$ of $\mathrm{ABS}$ content (cut from the core area of injection molded specimen, perpendicular view to flow): (a) $\mathrm{PSF} / \mathrm{PC}=85 / 15$; (b) $\mathrm{PSF} / \mathrm{PC}=70 / 30$; (c) $\mathrm{PSF} / \mathrm{PC}=50 / 50$; (d) $\mathrm{PSF} / \mathrm{PC}=30 / 70$ 

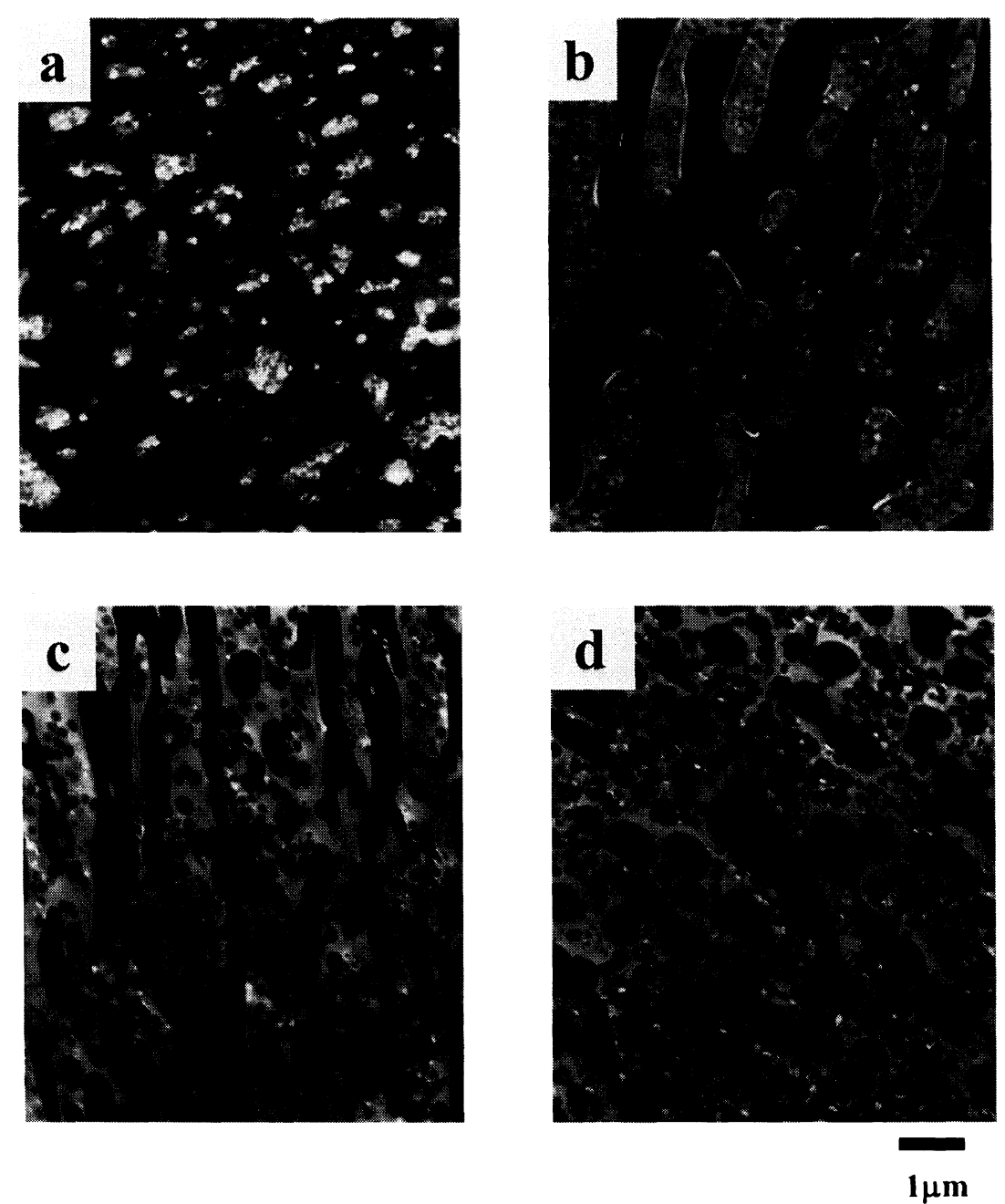

Figure 3. TEM photographs of $\mathrm{PSF} / \mathrm{PC} / \mathrm{MBE}$ blends with $15 \mathrm{wt} \%$ of $\mathrm{MBE}$ content (cut from the core area of injection molded specimen, perpendicular view to flow): (a) $\mathrm{PSF} / \mathrm{PC}=85 / 15$; (b) $\mathrm{PSF} / \mathrm{PC}=70 / 30$; (c) $\mathrm{PSF} / \mathrm{PC}=50 / 50$; (d) $\mathrm{PSF} / \mathrm{PC}=30 / 70$.

D256.

\section{RESULTS AND DISCUSSION}

Figure 2 shows the TEM micrographs of PSF/PC/ABS ternary blends. Although the $\mathrm{PSF} / \mathrm{PC}$ ratios are varied from $85 / 15$ to $30 / 70$, the content of ABS is fixed at $15 \mathrm{wt} \%$ of the blends. As benzene rings in polymer chains are preferentially stained with $\mathrm{RuO}_{4}$ vapor, ${ }^{10}$ it is important to designate which part in the micrographs corresponds to which polymers. Figure $2 b$, which is for PSF matrix blend, may give information on the contrast difference between PSF and PC. It is clear that PSF is more stained than PC under the staining condition applied in this study and thus appears darker. ABS domains exist in agglomerate form and appear gray. As shown in Figure 2 , morphology of the blends is very dependent on the matrix type. For PSF matrix (Figures $2 \mathrm{a}$ and $2 \mathrm{~b}$ ) PC is situated in the interface between PSF and ABS and forms encapsulating layer. Whereas, for PC matrix (Figures 2c and 2d) PSF and ABS have the tendency to exist in pure dispersed phases and any encapsulation is not observed. It is noteworthy that the PC phases located at interfaces do not contribute to break-up of agglomerate of ABS and pure PC phases also exist in PSF matrix.

Figure 3 shows the TEM micrographs of PSF/PC/
MBE ternary blends. The same compositions as Figure 2 are applied. The morphology shown in Figure 3 has similar tendency to Figure 2: encapsulation by PC onto MBE in PSF matrix and two distinct PSF and MBE phase in PC matrix. From the morphology observed we conclude that $\mathrm{PC}$ has the strong tendency to encapsulate ABS or MBE in PSF matrix. When PC was used as matrix PSF and ABS (or MBE) exists as distinct dispersed phases. The addition of PC does not contribute to effectively break-up of IM agglomerates for both blend series.

To verify whether the encapsulation observed in Figures 2 and 3 is thermodynamically favorable or not, ternary blends of PSF/PC/SAN24 and PSF/PC/PMMA are investigated because $\mathrm{SAN} 24$ and PMMA correspond to the shell of ABS and MBE, respectively. PSF is used as matrix for both cases. As shown in Figure 4 it is not difficult to distinguish each phase in the blends from blend compositions because SAN24 and PMMA have smallest amount for both blends. Figure 4 clearly shows that PC encapsulates SAN24 (Figure 4a) and PMMA (Figure 4b) in PSF matrix for both blends, indicating that during melt mixing $\mathrm{PC}$ has strong tendency to encapsulate the SAN or PMMA shells of ABS or MBE, respectively. It is thought that core polymers of $\mathrm{ABS}$ or MBE do not affect the encapsulation due to the cross- 

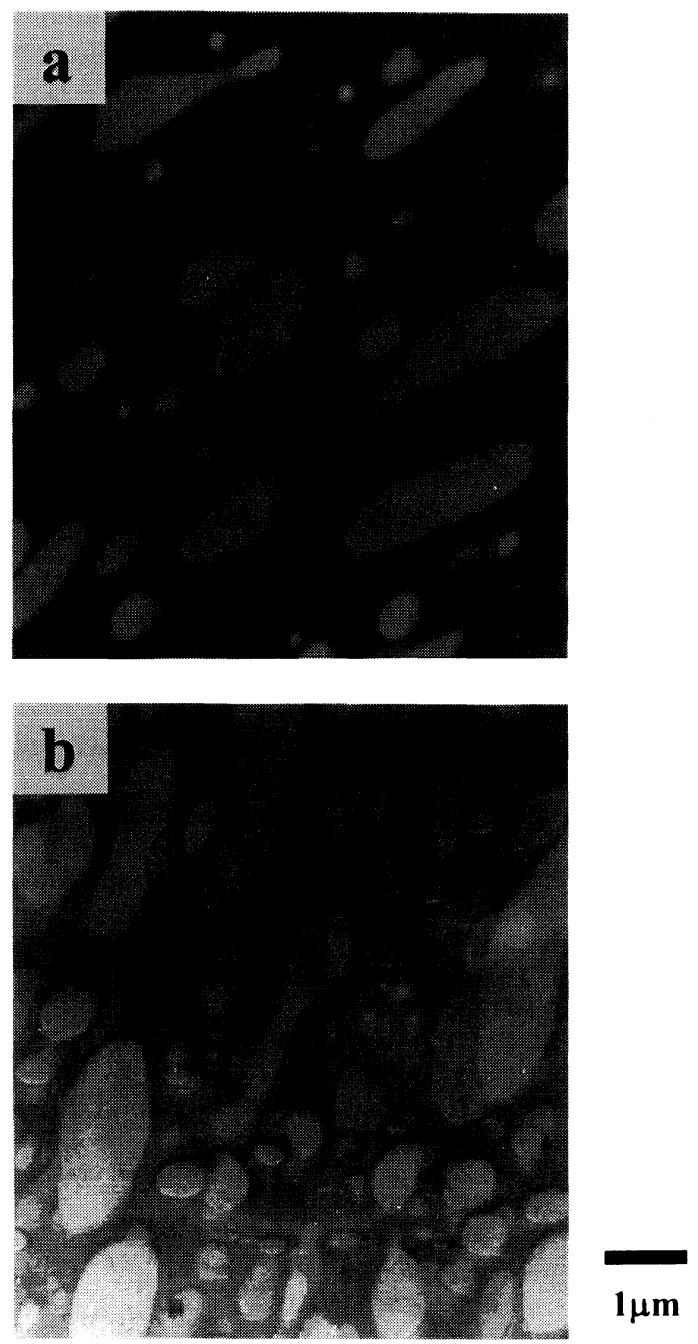

Figure 4. TEM photographs after mixing at a Haake batch mixer with rotor blades at $270^{\circ} \mathrm{C}$ and $50 \mathrm{rpm}$ for $10 \mathrm{~min}$ : (a) PSF $/ \mathrm{PC} / \mathrm{SAN} 24=$ 63/27/10; (b) PSF $/ \mathrm{PC} / \mathrm{PMMA}=63 / 27 / 10$.

\section{linked nature of them.}

The morphology observed in this study may be explained by the encapsulation model proposed by Hobbs and coworkers for ternary polymer blends. ${ }^{11}$ In the model, the third component moves to the interface and encapsulates the minor phase when the sum of the interfacial tensions associated with the third component, is smaller than the interfacial tension between the original blend components, i.e., $\gamma_{12} \geq \gamma_{13}+\gamma_{23}$, where component 1 is a matrix, component 2 is a minor phase and component 3 is the polymer added as encapsulating agent. However, it is not possible to quantitatively evaluate encapsulation behavior observed in this study because some of the interfacial tension data for the blend pairs are not available at this time. We simply postulate from the morphology of Figures 2 to 4 that $\gamma_{\mathrm{PSF} / \mathrm{SAN} 24}>\gamma_{\mathrm{PC} / \mathrm{SAN} 24}+\gamma_{\mathrm{PC} / \mathrm{PSF}}$ or $\gamma_{\mathrm{PSF} / \mathrm{PMMA}}>\gamma_{\mathrm{PC} / \mathrm{PMMA}}+$ $\gamma_{\mathrm{PC} / \mathrm{PSF}}$.

We may calculate $\gamma_{\mathrm{ij}}$ and thus $\lambda_{\mathrm{ij}}$ directly from surface tension data, as used by Hobbs et al. ${ }^{11}$ However, this approach has some difficulties; the parameters such as surface tension and its temperature gradient, which are necessary for calculation, has some error. The morphology observed here may be controlled by kinetic factors such as melt viscosity of blend components because low

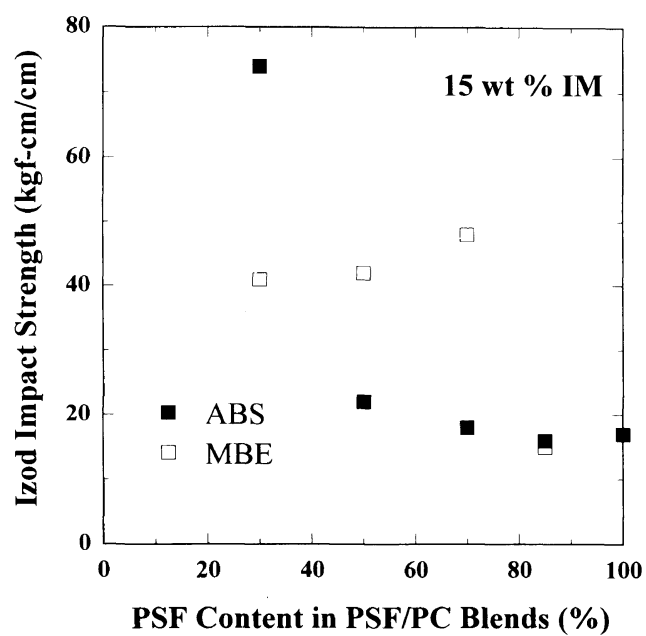

Figure 5. The effect of blend ratio of PSF/PC on notched Izod impact strengths of $1^{\prime \prime} / 4$ thickness specimen for PSF/PC/IM blends with $15 \mathrm{wt} \%$ of IM.

viscosity melts tend to encapsulate high viscosity melts during flow. Nemirovski et al. have insisted that the viscosity ratio of the two different minor phase is also important for developing encapsulated structure of immiscible ternary blends. ${ }^{12}$ They explained that when the viscosity of encapsulating polymer, which is predicted from spreading coefficient, is higher than that of the other, encapsulation is limited by high viscosity. However, when the viscosity ratio of dispersed and encapsulating polymers is not large, it seems not to be dominant factor for encapsulation. Recently, Lee et al. reported that for PS/PMMA/SMMA blends, where SMMA is a random copolymer of styrene and methyl methacrylate, SMMA moves to the interfaces during melt mixing to reduce the interfacial energy of the blends even though it has the highest melt viscosity under the mixing conditions employed. ${ }^{13}$ As shown in Figure 1, the melt viscosities of $\mathrm{ABS}$ and $\mathrm{MBE}$ have the lower value than that of PC, an encapsulating polymer at a frequency range of $100 \mathrm{~s}^{-1}$ or over, comparable to the shear rate of the mixing condition employed. Therefore, it is thought that the morphology observed here is controlled by thermodynamic factors such as interfacial tension.

Figure 5 shows the variation of notched Izod impact strength with the relative ratio of $\mathrm{PSF} / \mathrm{PC}$ for both blend series. The content of IM is fixed at $15 \mathrm{wt} \%$. Without $\mathrm{PC}$, impact strengths of PSF/MBE and PSF/ABS blends are very low, indicating that $\mathrm{ABS}$ and $\mathrm{MBE}$ used in this study are not effective in improving the impact strength of PSF. However, an increase of impact strength is observed when specific compositions of PSF and PC are applied; at $30 \mathrm{wt} \% \mathrm{PC}$ for MBE and $70 \mathrm{wt} \%$ for ABS containing blends, respectively. Blends with $\mathrm{MBE}$ show the increase in impact strengths when PC encapsulates MBE particles. Whereas, for ABS containing blends, the composition at which impact strength increases does not coincide with the morphology showing encapsulation. The maximum impact strength is observed when two distinct ABS and PSF phases exist in PC matrix. Higher impact strengths with more than $50 \mathrm{wt} \%$ of $\mathrm{PC}$ may be the result of matrix inversion: at $50 / 50 \mathrm{PSF} / \mathrm{PC}$ blend $\mathrm{PC}$ acts as matrix as shown in Figures $2 \mathrm{c}$ and $3 \mathrm{c}$. It is 
known that $\mathrm{PC}$ is usually well toughened by $\mathrm{MBE}$ or ABS. ${ }^{14}$ This result shows that the encapsulating layer of PC does not always contribute to improve the mechanical properties of the blends studied here.

In polymer blends, it is critical to establish some level of interfacial adhesion between blend components in order to achieve the necessary toughness and delamination resistance. ${ }^{15}$ This has been successfully achieved by adding block or graft copolymers as compatibilizers. ${ }^{16,17}$ Homopolymers which tend to encapsulate dispersed phases may also be considered as potential interface modifiers because they reduce the interfacial tension as explained by Hobbs et al. ${ }^{11}$ Reduction of interfacial tension results in the increase in interfacial adhesion, which is proportional to the interfacial thickness. The effectiveness of encapsulating polymers may be found from the results from ref 18 to 21 . However, it is still under controversy whether encapsulating layer of third homopolymers contributes to improve the interfacial properties of the polymer blends. Noolandi insists that although encapsulation by third polymers contributes to reduce the interfacial tension of original blends, the interfaces are different from those modified by graft or block copolymers and are expected to be weak. ${ }^{22}$

Blends of PC/SAN24 and PC/PMMA are compatible and thus the interfaces between $\mathrm{PC} / \mathrm{ABS}$ and $\mathrm{PC} / \mathrm{MBE}$ are not weak. ${ }^{23,24}$ Therefore, the toughness of the ternary blends studied here is governed by the interfaces between PSF and PC. Since PSF and PC are incompatible their interface is assumed to be very weak, resulting in poor mechanical properties. The blend of $30 / 70 \mathrm{PSF} / \mathrm{PC}$ has the value of impact strength less than $5 \mathrm{kgf} \mathrm{cm} \mathrm{cm}^{-1}$. To quantitatively compare the effectiveness of ABS and MBE we have to consider the factors such as the nature of rubber, content of rubbers, and particle size, which are critical for rubber toughening of thermoplastics. Therefore, although MBE used in this study seem to be more effective than ABS from the impact strength results, it cannot be said conclusively that $\mathrm{MBE}$ be more effective than ABS for the toughening of the PSF/PC blends.

\section{SUMMARY}

In order to improve the mechanical properties of immiscible PSF/PC blends two different core-shell impact modifiers, ABS and $\mathrm{MBE}$, were added. Irrespective to IM type, PC has the tendency to encapsulate IM when PSF acts as matrix. For PC matrix blends two distinct PSF and IM phases are observed. However, even though PC encapsulates the IM it does not effectively break-up agglomerates of IM particles and enhances the impact strengths of the blends, especially, for ABS containing blends.

Acknowledgments. MSL wishes to acknowledge the partial financial support of Chonnam National University made in the program year of 1997.

\section{REFERENCES}

1. J. A. Brydson, "Plastics Materials," 5th ed, Butterworths, London, 1989.

2. D. Debier, J. Devaux, and R. Legras, Polym. Eng. Sci., 34, 613 (1994).

3. J. Wu, Y. W. Mai, and B. Cotterell, J. Mater. Sci., 28, 3373 (1993).

4. F. S. Myers and J. O. Brittain, J. Appl. Polym. Sci., 17, 2715 (1973).

5. M. M. Kerber and Y. K. Godovskii, Vysokomol. Soedin., Ser. A, 35, 808 (1993)

6. W. G. Kohlman and S. P. Petrie, Adv. Polym. Tech., 14, 111 (1995).

7. D. R. Paul and S. Newman, Ed., "Polymer Blends," Academic Press, New York, N.Y., 1978.

8. C. B. Bucknall, "Toughened Plastics," Applied Science Publishers, London, 1977.

9. S. Hosoda and K. Kojima, Polym. Commun., 30, 83 (1989).

10. J. S. Trent, J. I. Scheinbeim, and R. R. Couchman, Macromolecules, 16, 589 (1983).

11. S. Y. Hobbs, M. E. J. Dekkers, and V. H. Watkins, Polymer, 29, 1598 (1988)

12. N. Nemirovski, A. Siegmann, and M. Narkis, J. Macromol. Sci.-Phys., B34, 459 (1995).

13. M. S. Lee, T. P. Lodge, and C. W. Macosko, J. Polym. Sci., Polym. Phys., 35, 2835 (1997).

14. D. S. Parker, H-J. Sue, J. Huang, and A. F. Yee, Polymer, 31, 2267 (1990)

15. S. Wu, Polym. Eng. Sci., 27, 335 (1987).

16. H. R. Brown, Annu. Rev. Mater. Sci., 21, 463 (1991).

17. S. Datta and D. J. Lohse, "Polymeric Compatibilizers," Hanser Publishers, Munich, 1996.

18. M. E. J. Dekkers, S. Y. Hobbs, and V. H. Watkins, Polymer, 32, 2150 (1991).

19. A. J. Brady, H. Keskkula, and D. R. Paul, Polymer, 35, 3665 (1994).

20. S. Horiuchi, N. Matchariyakul, K. Yase, T. Kitano, H. K. Choi, and Y. M. Lee, Polymer, 38, 6317 (1997).

21. S. Horiuchi, N. Matchariyakul, K. Yase, T. Kitano, H. K. Choi, and Y. M. Lee, Polymer, 38, 59 (1997).

22. J. Noolandi, "Polymeric Materials Encyclopedia," Vol. 9, J. C. Salamone, Ed., CRC Press, New York, N.Y., 1996.

23. R. P. Wool, "Polymer Interfaces," Hanser Publishers, Munich, 1995, Chapter 9.

24. M. Nishimoto, H. Keskkula, and D. R. Paul, Polymer, 32, 272 (1991). 